\title{
Unilateral cataract: A potentially fatal prognosis
}

Ju-Lee Ooi, Michelle Mei Pik Hui, Neil S Sharma, Michael Giblin, Georgina Clark

\section{CASE}

A man aged 67 years was referred by his general practitioner (GP) for assessment of a right cataract. He had noticed visual blurring over the preceding four weeks. On examination, the right visual acuity (VA) was 6/9-2 and left VA was 6/7.5. The right eye had a localised posterior subscapular cataract located in the superior part of the crystalline lens in close proximity to the visual axis. Dilated fundus examination revealed a large pigmented mass superiorly (Figure 1).

\section{Question 1}

What are the differential diagnoses for unilateral cataract?

\section{Question 2}

What is ciliochoroidal melanoma?

\section{Question 3}

What further investigations would confirm the diagnosis?

\section{Answer 1}

Deterioration of vision and myopic shift in people aged over 60 years is often attributed to cataract by primary care providers. Predisposing factors to bilateral cataracts include prolonged exposure to ultraviolet light, smoking and diabetes. Unilateral cataract can be due to less sinister causes such as inflammation, infection and trauma, or to more sinister causes such as tumours. This presentation was initially mistaken by the GP as being solely due to cataract.

The challenges in diagnosis include that patients are often asymptomatic or present with non-specific symptoms such as blurred vision or floaters. ${ }^{1-3}$ Unfortunately, 28-37\% of uveal melanomas were missed in the first examination, leading to a delay in the diagnosis. ${ }^{1-3}$ It is important to consider increasing the urgency of referral to an ophthalmologist if the case for cataract is not completely convincing, especially if there is severe asymmetry in vision, or if there are symptoms such as metamorphopsia or photopsia, which suggest a posterior segment pathology. The challenge for the GP is that waiting times for cataract clinics can be up to one year in some public hospitals. ${ }^{4}$ Given such delays, referrals to optometrists for verification of the diagnosis with a dilated examination are an option.

\section{Answer 2}

Ciliochoroidal melanoma is a type of uveal melanoma. The uvea is a dense, pigmented layer between the sclera and retina, which subdivides into the iris, ciliary body and choroid. More than $85 \%$ of uveal melanomas arise in the choroid, while the remainder arise in the iris (2\%) or ciliary body (7\%). ${ }^{1-3,5}$ In Australia, the prevalence of choroidal melanoma for both sexes is estimated to be 7.2 per million, close to double that in the United States and Europe. ${ }^{1,2,5}$

Risk factors linked to uveal melanoma includes age (65 years and older); light iris pigmentation; fair skin; preexisting choroidal nevus; dysplastic naevus syndrome and familial uveal melanoma. $^{1-3,5}$

\section{Answer 3}

The diagnosis is primarily detected on dilated fundus examination. The classic appearance is a pigmented, dome-shaped subretinal mass with associated subretinal fluid. ${ }^{1-3}$ On examination of the red reflex, a dome-shaped shadow may be seen instead of the classical wedge shadow of a cortical cataract or an overall dull reflex from a nuclear cataract. Sentinel blood vessels may be visible.

Once confirmed, staging of the disease requires serum tests (complete blood count and liver function tests [LFTs]) and imaging (magnetic resonance imaging [MRI] of liver and whole-body positron emission tomography/computer tomography). Current guidelines recommend annual follow-up consisting of clinical assessment with LFTs, MRI of the abdomen and/or ultrasound of theliver and chest radiograph indefinitely. ${ }^{2}$

\section{CASE CONTINUED}

B-scan ultrasonography revealed a right superior ciliochoroidal melanoma with a base measuring $15 \mathrm{~mm}$ and a thickness of $6.9 \mathrm{~mm}$ (Figure 2). The ciliochoroidal melanoma was treated with plaque radiotherapy via direct irradiation of the tumour with the application of radioisotope (Iodine-125). On follow-up, the tumour thickness had shrunk to $5 \mathrm{~mm}$ and is subject to ongoing monitoring.

\section{Question 4}

What are the treatment and prognostic factors?

\section{Answer 4}

For patients with no metastasis, Singh et al observed that from 1973 to 2008 , the 


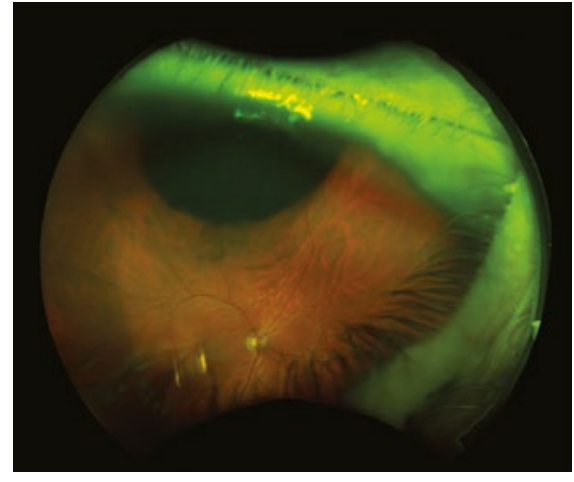

Figure 1. Dilated fundus examination revealed a large pigmented mass superiorly

five-year survival rate remained similar at $81.6 \%$ with no significant difference between the mortality in those who had enucleation or an eye-conserving approach. ${ }^{3}$ Therefore, in recent years there has been a move towards more eye-conserving local therapies. ${ }^{1-3}$ Local treatments are effective in preventing recurrence in more than $95 \%$ of cases. These treatments include transpupillary thermotherapy, proton beam therapy, surgical excision, photocoagulation and, most commonly, radiotherapy., ${ }^{1,2}$

The five-year metastasis rates in patients with choroidal melanoma were $25 \%$, as observed in the Collaborative Ocular Melanoma Study, irrespective of the tumour size. ${ }^{6}$ The liver (93\%) is the most commonly observed site of metastases. ${ }^{1,6}$ Unfortunately, the longterm prognosis of metastatic disease is poor, with a one-year survival rate at $10-15 \% .{ }^{1-3,6}$ A new immunotherapy, pembrolizumab, showed hope with significantly prolonged overall survival in patients with metastatic melanoma; however, further long-term studies are required. ${ }^{7,8}$

\section{Key points}

- Presentations of unilateral cataract in middle-aged and older patients (40-80 years) should prompt consideration of posterior segment pathology, including ocular melanoma. These patients require timely referral to an

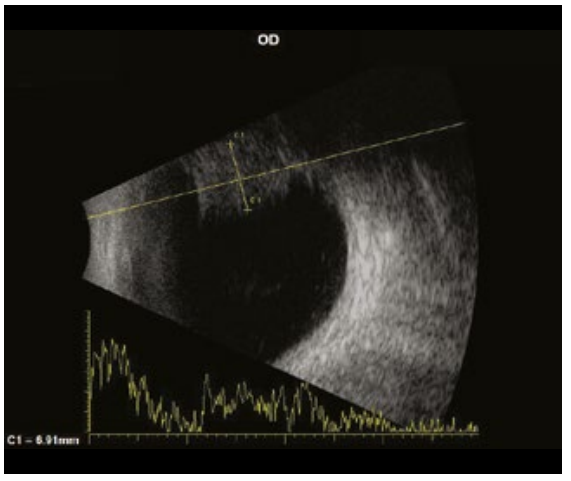

Figure 2. B-scan ultrasonography demonstrated a $6.9 \mathrm{~mm}$ thick hyper-echoic ciliochoroidal melanoma in the right superior quadrant

ophthalmologist for dilated fundal examination.

- Uveal melanoma is primarily diagnosed clinically via dilated fundoscopy. The classic feature on dilated fundoscopy is a pigmented dome-shaped or mushroom-shaped mass.

- Early diagnosis of uveal melanoma is crucial, given the risk of metastases and a grim prognosis once metastasis occurs, with a one-year survival rate of $10-15 \%$.

- Uveal melanoma is the most common type of ocular melanoma; choroidal melanoma accounts for $85 \%$ of uveal melanoma. In Australia, the prevalence of uveal melanoma is close to double that in Europe and the United States.

\section{Authors}

Ju-Lee Ooi BSc(Med), MBBS, MPH, GradDipPaed, FRANZCO, Ophthalmologist and Medical Retina Specialist, Eye and Retina Specialists, Green Square, Sydney, NSW; Consultant Ophthalmologist, Westmead Hospital, Sydney, Clinical Lecturer, University of Sydney, Conjoint Lecturer, University of New South Wales, NSW. juleeooi@hotmail.com

Michelle Mei Pik Hui BMed, MD, Senior Resident Medical Officer, Prince Alfred Hospital, Sydney, University of Sydney, Medicine, Sydney, NSW Neil S Sharma BSc (Med), MBBS (Hons), MPH, MMed (Refract Surg), FRANZCO, Ophthalmologist and Medical Retina Specialist, Eye and Retina Specialists, Green Square, Sydney, NSW; Consultant Ophthalmologist, Westmead Hospital, Senior Clinical Lecturer, University of Sydney; Senior Clinical Lecturer, University of Wollongong, NSW

Michael Giblin MBBS (Hons), FRANZCO, FRACS, Consultant Ophthalmologist, Sydney Eye Hospital, Sydney, NSW, and University of Sydney, Medicine, Sydney, NSW
Georgina Clark MBBS, FRANZCO, Medical Retina and Uveitis Fellow, Bristol University Hospitals Trust, NHS Foundation Trust, Bristol, UK, University of Sydney, Medicine, Sydney, NSW

Competing interests: None.

Provenance and peer review: Not commissioned, externally peer reviewed.

\section{References}

1. Tarlan B, Kıratlı H. Uveal melanoma: Current trends in diagnosis and management. Turk Ophthalmol 2016;46(3):123-37.

2. Weis E, Salopek TG, McKinnon JG, et al. Management of uveal melanoma: A consensusbased provincial clinical practice guideline. Curr Oncol 2016;23(1):e57-64. doi: 10.3747/co.23.2859.

3. Singh AD, Turell ME, Topham AK. Uveal melanoma: Trends in incidence, treatment, and survival. Ophthalmology 2011;118(9):1881-85. doi:10.1016/j.ophtha.2011.01.040.

4. Alexander $\mathrm{H}$. Cataract surgery patients languish on hidden waiting list for elective hospital procedures. Sydney Morning Herald. 10 June 2015.

5. Vajdic CM, Kricker A, Giblin M, et al. Incidence of ocular melanoma in Australia from 1990 to 1998. Int J Cancer 2003;105(1):117-22.

6. Diener-West M, Reynolds SM, Agugliaro DJ, et al. Development of metastatic disease after enrollment in the COMS trials for treatment of choroidal melanoma: Collaborative Ocular Melanoma Study Group Report No. 26. Arch Ophthalmol 2005;123(12):1639-43.

7. Robert C, Schachter J, Long GV, et al. Pembrolizumab versus ipilimumab in advanced melanoma. N Engl J Med 2015;372(26):2521-32. doi: 10.1056/NEJMoa1503093.

8. Watson I, Dominguez PP, Donegan E, Charles Z, Robertson J, Adam EJ. NICE guidance on pembrolizumab for advanced melanoma. Lancet Oncol 2016;17(1):21-22. doi: 10.1016/S14702045(15)00547-1. 